\title{
Apparel: From reality to virtual reality
}

\author{
Jean-Marc SURVILLE \\ Lectra (France)
}

\begin{abstract}
In this session we explain how to become confident in the virtual garment simulation.

The process is based on a parallel session for creating a real garment on a real person alive, and then to simulate the same garment on her 3D avatar using Modaris 3D Fit, Lectra virtual prototyping solution. A person has been scanned using a body scanner, and then introduced into the virtual world to process a virtual try-on on her own body.

The parallel development of the garment on the real body and on her avatar enhances how to judge the fitting of the garment. Results show how defects in garment construction can be detected as part of a virtual prototyping process, compared to the physical samples and relating to the clothing skill.

The user then becomes confident into the virtual try on as a professional tool

A symmetric process then takes place. The user develops a style, using Modaris, the 2D lectra software, simulates the try-on in the virtual environment of Madaris 3DFit before deciding to cut and assemble the physical garment and finally try it on.Benefits to the development of prototypes can be evaluated.As a result this session claims to belong to an industrial virtual prototyping.
\end{abstract}

Keywords: virtual garment, scanned body, garment fitting, training

\section{Introduction,}

The introduction of 3D virtual try on in the market reveals different approaches. For garment fitting, as well as garment construction using the 3D mannequin body, the clothing industry needs to take this new world to its own.

In this session we explain how to become confident in the virtual garment simulation.

The process is based on a parallel session for creating a real garment on a real person alive, and then to simulate the same garment on her 3D avatar using Modaris 3D Fit, Lectra virtual prototyping solution.

\section{On the way to virtual world}

\subsection{Preparing the try-on}

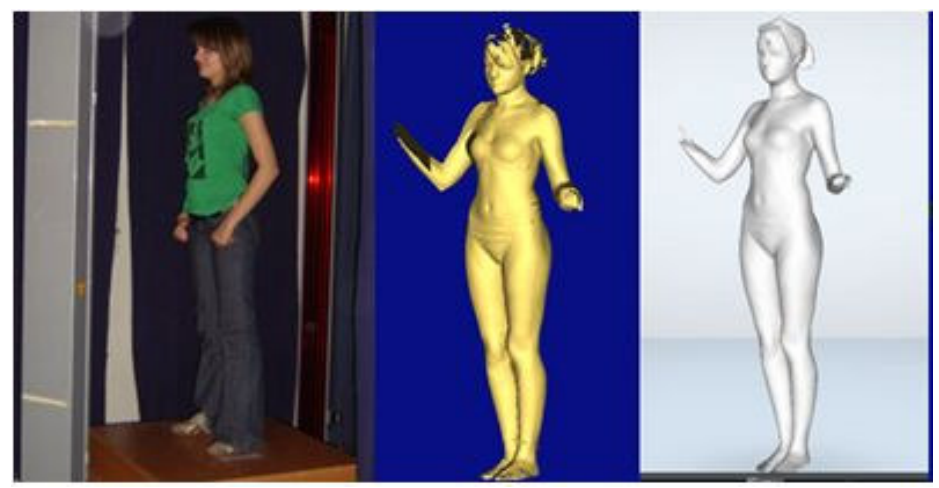

Figure 1 process to enter virtual world

The students have been scanned using a body scanner, and then introduced into the virtual world to process a virtual try-on on their own bodies 


\subsection{Basic skirt construction}

The students used a construction based on their measurements taken on their own scanned bodies Ease was evaluated according to the style decided.(1)

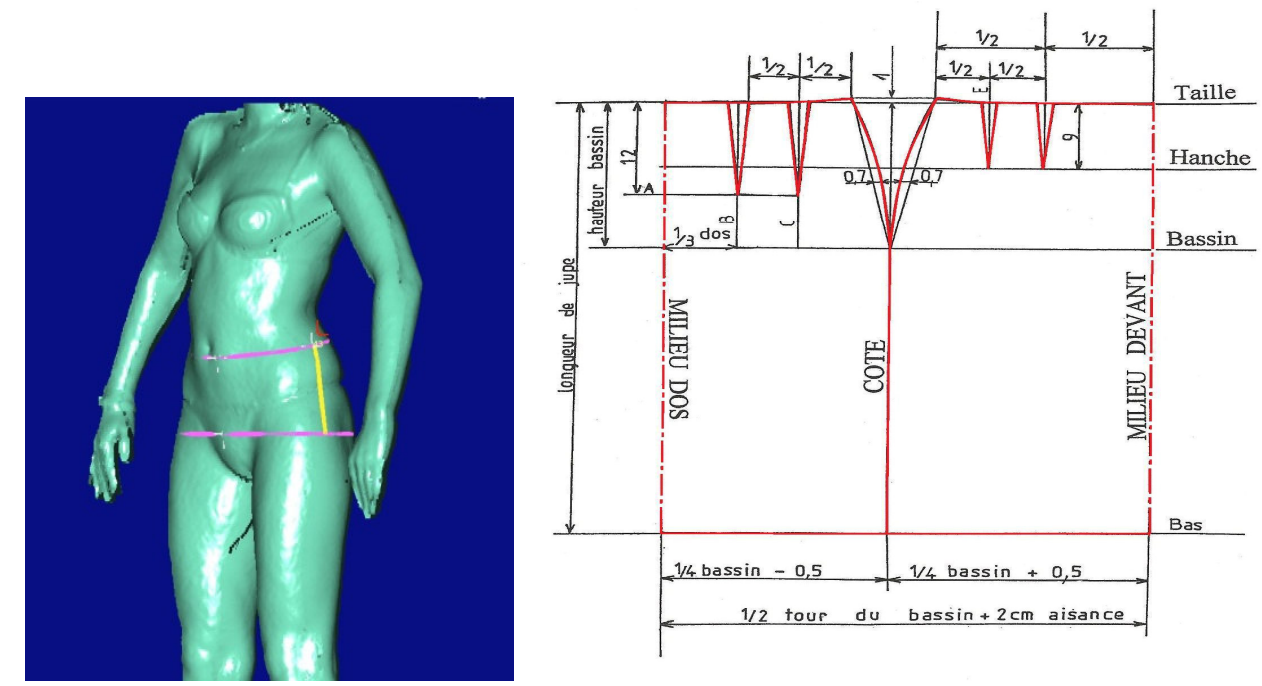

Figure 2 basic construction using body measurements

The construction is realized according to the student skill academic references (Aldrich), in fact a manual construction on paper

Then the skirt is digitized through the Modaris $2 \mathrm{D}$ lectra program

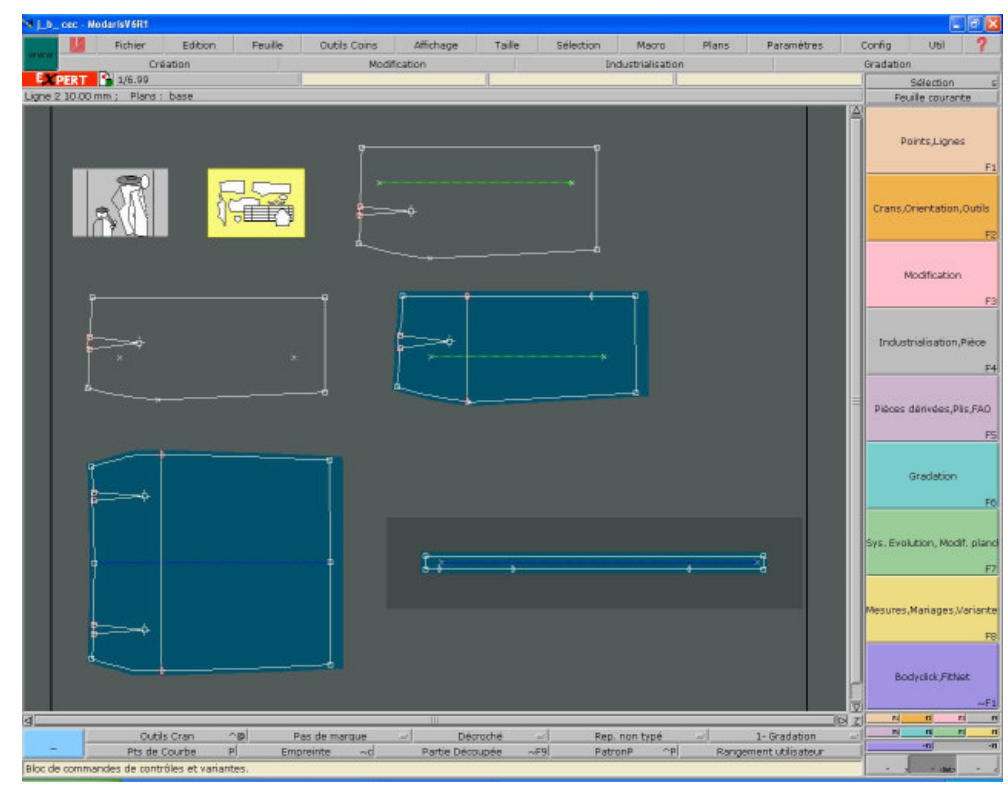

Figure 3 Modaris digitized pieces

\subsection{Production process}

The functions available in the Modaris program allow preparing the model for cutting, after seam allowance addition, and controls for the body measurement matching.

The cutting fabric and sewing are processed on industrial machines.

At this state of the process the students have a real skirt for each of them as well as the $2 \mathrm{D}$ models available in the software Modaris. 


\subsection{The try-on revelation.}

\subsubsection{The real skirts}

This try-on session was mainly dedicated to make the students understand the relationship between their body and the garment they personally built on their own body measurements.
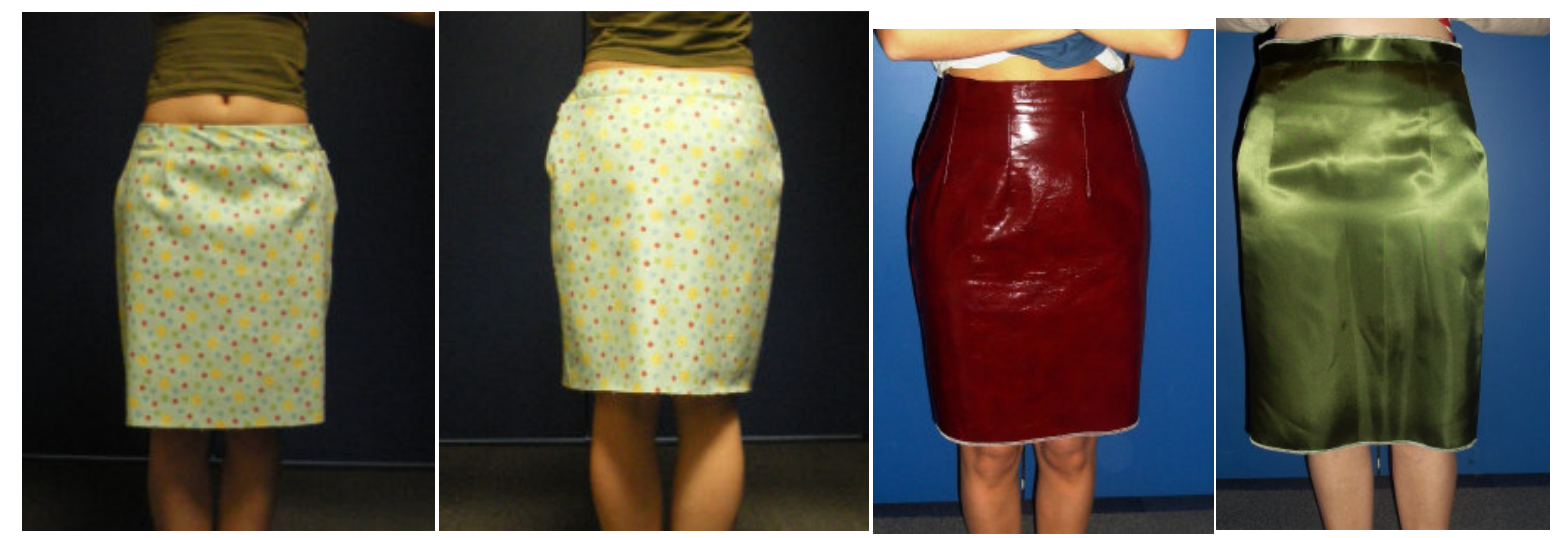

Figure 4 three students basic constructions

The analysis of the defaults were based on "standards for fitting a basic garment" ((2) p 58)

\subsubsection{Fitting a skirt}

A classical default was detected due to small waist and flat abdomen on young women.

They verified the pertinence and the fitting procedure on different documents (aldr, fairshild)

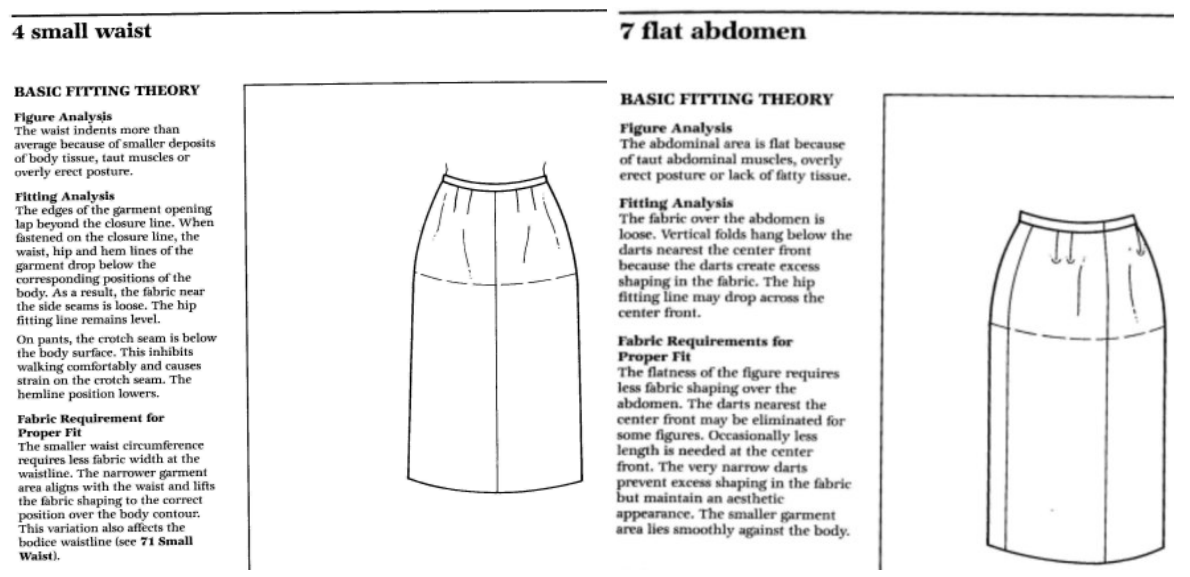

Figure 5 fitting problems detected (2)

Once this situation understood they went to the virtual world.

\subsection{Entering the virtual world.}

\subsubsection{The body scanner}

The real try-on was memorized as a scan. This allow the students to visit their $3 \mathrm{D}$ image as far as necessary to view the garment on their own body. 

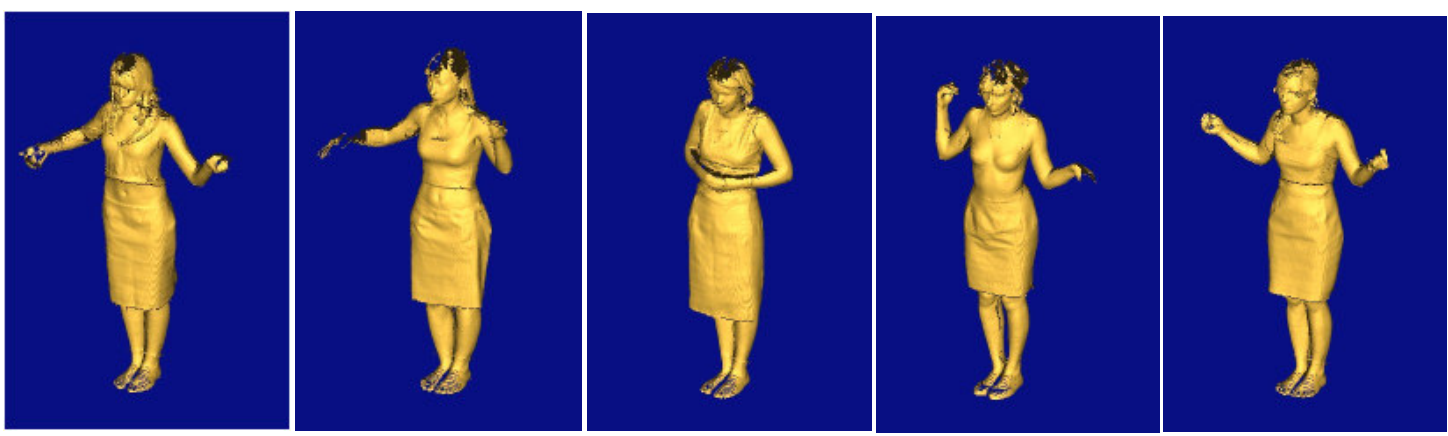

Figure 6 some student skirts scanned

The fitting aspects appear on each of them and then will be available for deeper analysis.

\subsubsection{Entering virtual try-on Modaris 3D Fit}

The scans were prepared according to the standards for the software Modaris 3D fit. All were exported as a vrml 1.0 version format.

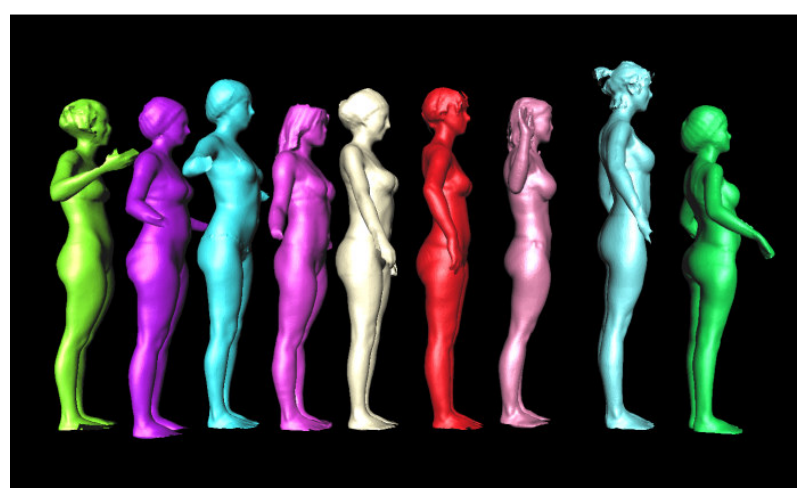

Figure 7 Some students prepared for virtual try-on

\subsubsection{Entering skirts into the virtual try-on world Modaris 3D-Fit}

Once the garment assembly proceeded, the first simulation of the skirt is proceeded.

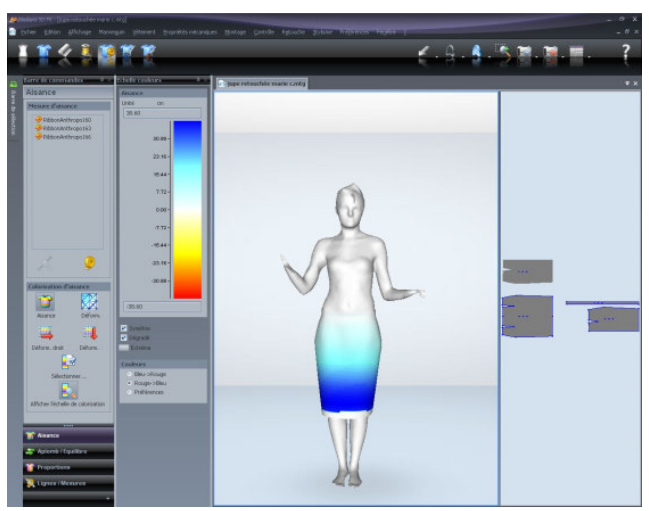

Figure 8 Modaris 3D fit environment 


\section{Test/Data,}

\subsection{Real virtual close analysis.}

The parallel development of the garment on the real body and the simulation of the skirt draping on her avatar enhances how to judge the fitting of the garment.

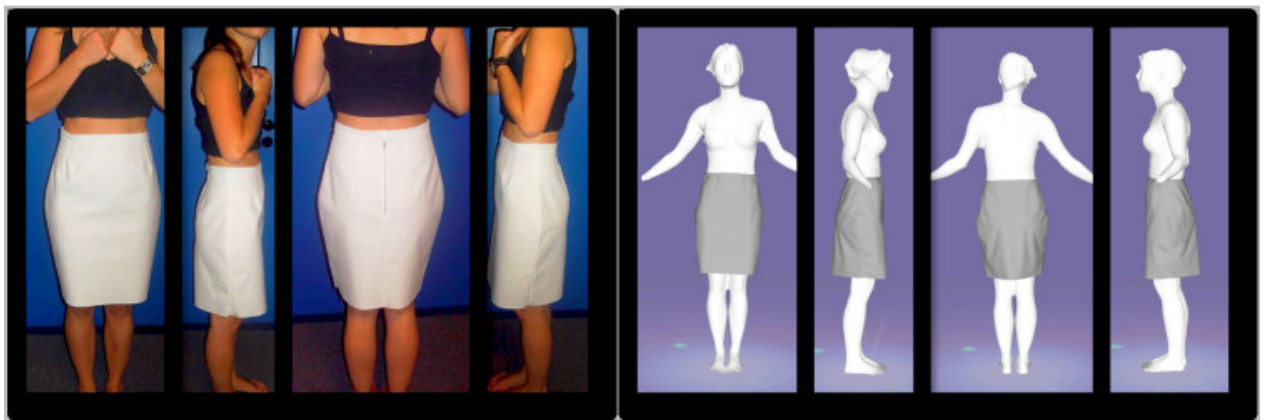

Figure 9 A student and her avatar.

The scenes show how defects in garment construction can be detected as part of a virtual prototyping process, compared to the physical samples and relating to the clothing skill

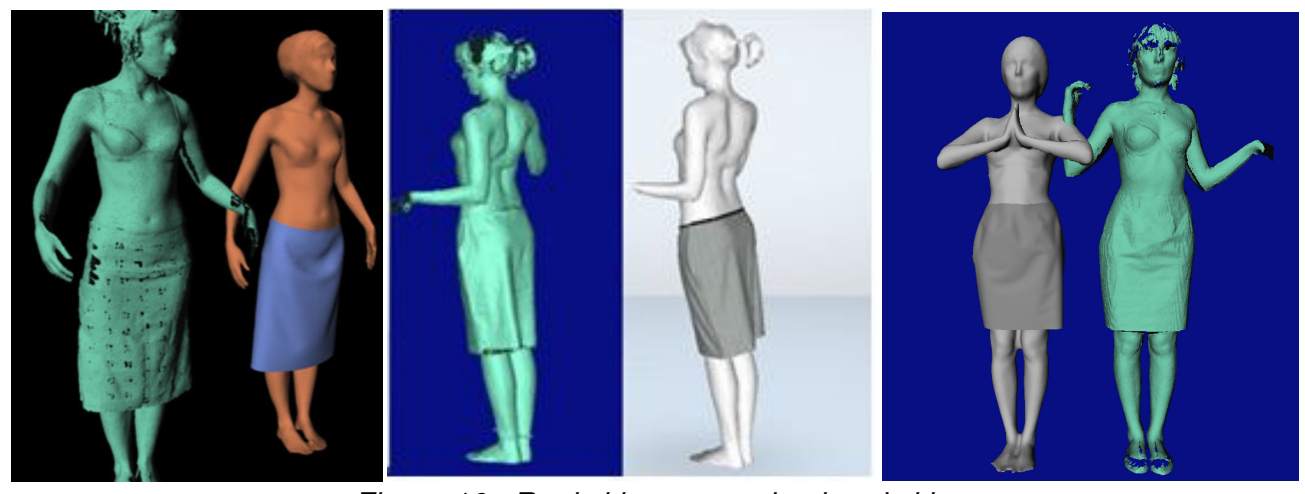

Figure 10. Real skirt versus simulated skirt

Understanding the body relations using transparency.

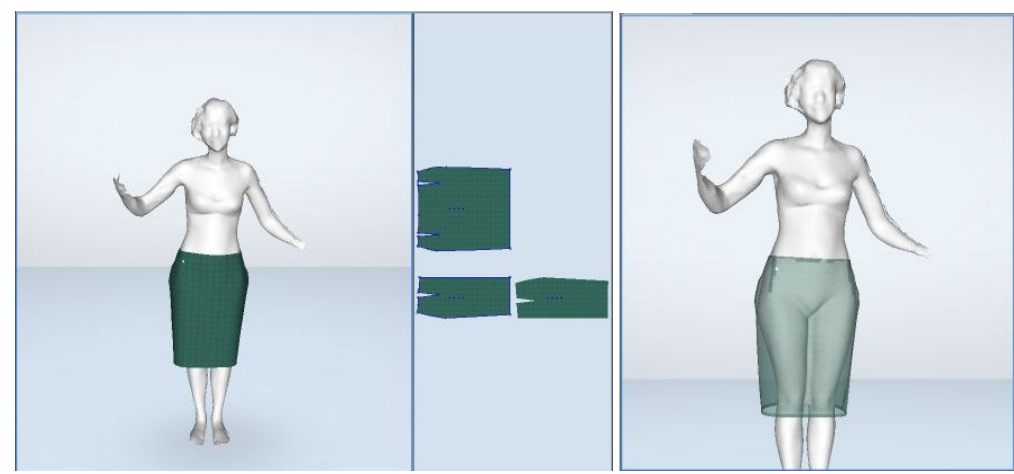

Figure 11 Effects by transparency.

The user then becomes confident into the virtual try on as a professional tool to evaluate the first tryon 


\section{2 modfication cycle in the virtual world.}

The flat abdomen effect appears both on real skirt as well as on simulated skirt.

The skill is an important part for teaching and understanding the behavior of the garment onto the body. Refering to the academic skill (see fig 5) a cycle in the virtual world is processed, alteration using Modaris 2D and immediate simulation with Modaris 3DFit.

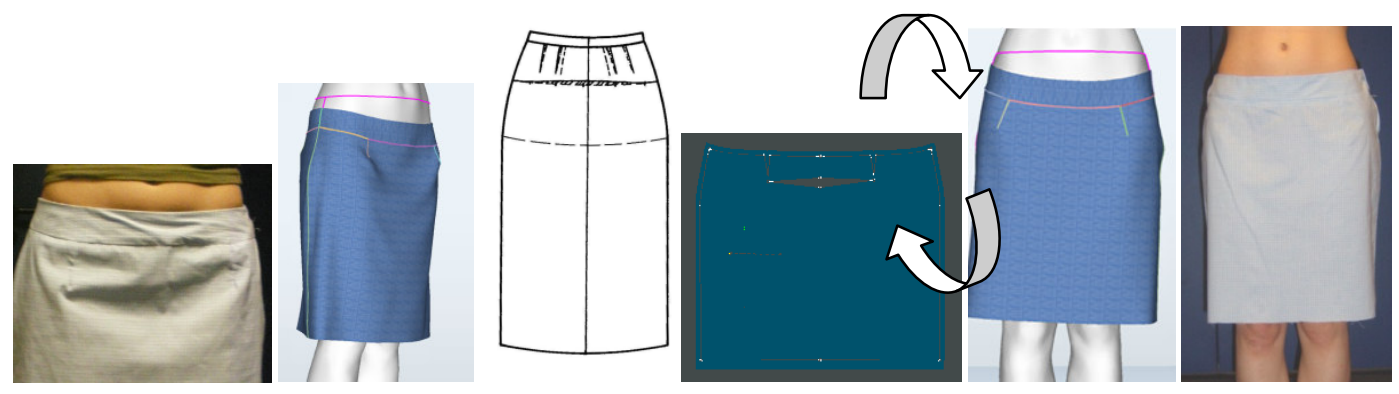

Figure 12 cycle for virtual alteration

During the successive iterations the student understands the garment behavior, and gets a confident attitude regarding virtual try-on. Then the corrected skirt is sewn and tried in real.

\section{Back to reality.}

A symmetric process then takes place. The user develops a style, using Modaris, the 2D lectra software, simulates the try-on in the virtual environment of Madaris 3DFit before deciding to cut and assemble the physical garment and finally try it on.

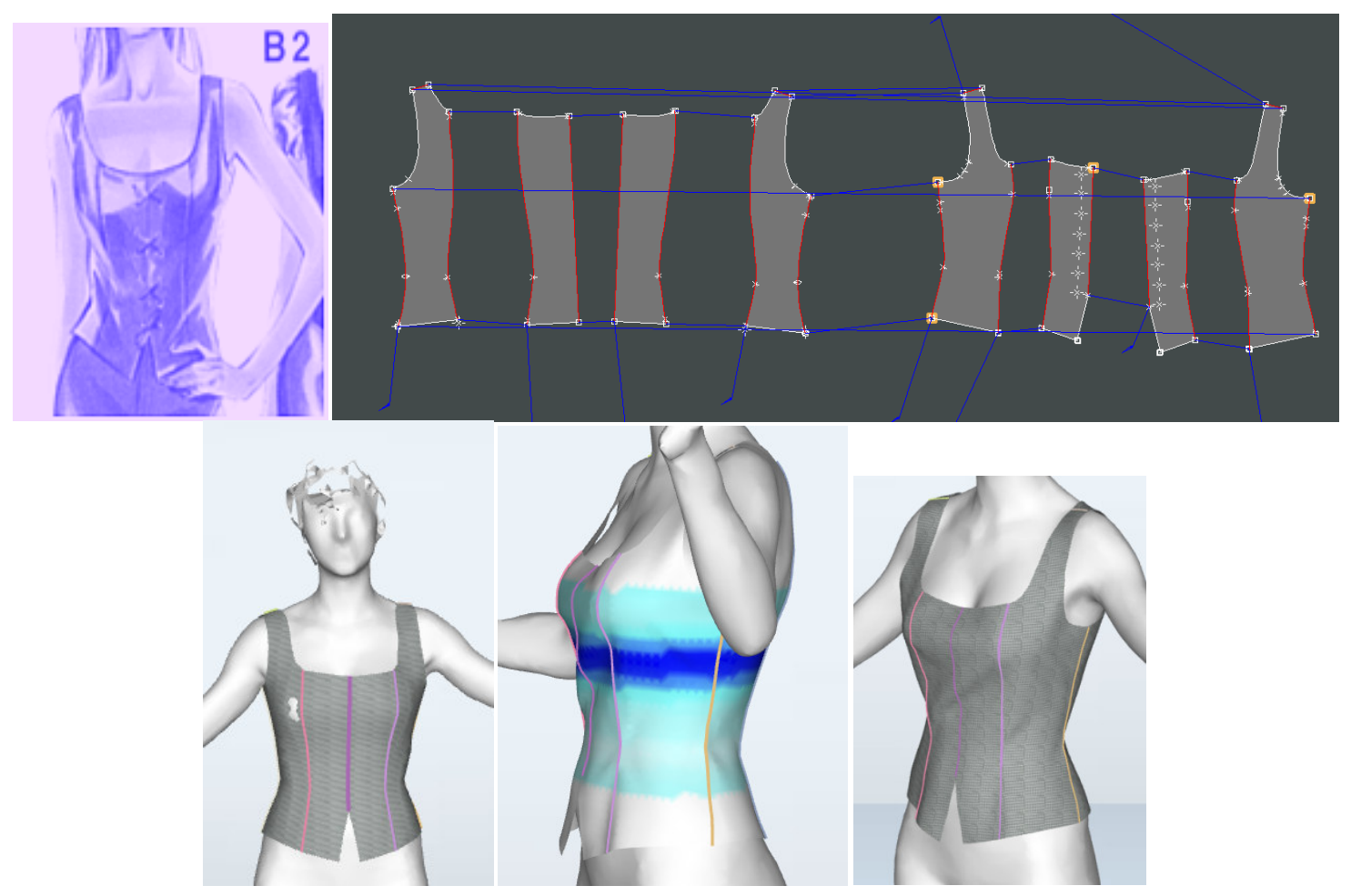

Figure 13 virtual development on the real body.

The tools available in the software such as transparency or ease .In the example above (fig 13) the software indicates that the under bust is too tight . 


\section{Discussion conclusion.}

Our approach for virtual try on includes two realist references. The real body of the students, and the real construction of a skirt based on clothing skill. This approach has been presented by some authors $(7,8)$. However our goal is the direct relationship between the body and the garment analyzed in the real and virtual world and not only a realistic representation.

In an industrial context, the user becomes an efficient actor, with a minimum tool required to enhance creativity. Benefits may be estimated as saving the first prototypes and the fast cycle to simulation and the real first prototype.

As a matter of teaching, this session claims to allow student understand the reality of wearing a garment, in an alive context, far away from the workshop mannequins.

\section{References}

(1) W. Aldrich, metric pattern cutting Wiley-Blackwell; 4th Edition edition (29 July 2004)

(2) Elizabeth L. Liechty, Della N. Pottberg-Steineckert, Judith A. Rasband, Fitting and Pattern Alteration: A Multi-Method Approach. 1992 New York Fairchild publications

(3) Catherine Black, Modaris, Diamino, and JustPrint for Apparel Design, . 2007 New York Fairchild publications

(4) Hein Daanen, Sung-Ae Hong, (2008) "Made-to-measure pattern development based on 3D whole body scans", International Journal of Clothing Science and Technology, Vol. 20 Iss: 1, pp.15-25

(5) Susan P. Ashdown, Suzanne Loker, Katherine Schoenfelder, and Lindsay Lyman-Clarke (2004) USING 3D SCANS FOR FIT ANALYSIS Journal of Textile and Apparel, Technology and Management 4,(1)

(6) Xiaoqun Dai, Takao Furukawa, Shigeru Mitsui, Masayuki Takatera, Yoshio Shimizu, (2001) "Drape formation based on geometric constraints and its application to skirt modelling", International Journal of Clothing Science and Technology, Vol. 13 Iss: 1, pp.23-37

(7) Susan Ashdown, Suzanne Loker, co-leaders (Cornell); Margaret Rucker (UC Davis) Improved Apparel Sizing: Fit and Anthropometric 3D Scan Data NTC Project: S04-CR01 http://www.ntcresearch.org/projectapp/?project=S04-CR01

(8) M. Wacker and Al., Simulation and Visualisation of Virtual Textilesfor Virtual Try-On Research Journal of Textile and Apparel Vol. 9 No. 12005

(9) Choong Hyo Kim, In Hwan Sul, Chang Kyu Park, Sungmin Kim, (2010) "Automatic basic garment pattern generation using three-dimensional measurements", International Journal of Clothing Science and Technology, Vol. 22 Iss: 2/3, pp.101-113

(10)Young Sook Cho, Keiichi Tsuchiya, Masayuki Takatera, Shigeru Inui, Hyejun Park, Yoshio Shimizu, (2010) "Computerized pattern making focus on fitting to 3D human body shapes", International Journal of Clothing Science and Technology, Vol. 22 Iss: 1, pp.16-24

(11) Elizabeth Bye, Ellen McKinney, (2010) "Fit analysis using live and 3D scan models", International Journal of Clothing Science and Technology, Vol. 22 Iss: 2/3, pp.88-100 